\title{
Fuzzy fixed point results of generalized almost F-contractions in controlled metric spaces
}

\author{
Nayab Alamgir ${ }^{1}$, Quanita Kiran ${ }^{2}$, Hassen Aydi $^{3,4,5^{*}}$ and Yaé Ulrich Gaba ${ }^{6 *}$
}

\author{
"Correspondence: \\ hassen.aydi@isima.rnu.tn; \\ yaeulrich.gaba@gmail.com \\ ${ }^{3}$ Institut Supérieur d'Informatique et \\ des Techniques de Communication, \\ Université de Sousse, \\ H. Sousse 4000, Sousse, Tunisia \\ ${ }^{6}$ Quantum Leap Africa (QLA), AIMS \\ Rwanda Centre, Remera Sector KN \\ 3, Kigali, Rwanda \\ Full list of author information is \\ available at the end of the article
}

\begin{abstract}
In this paper, we derive some common $\alpha$-fuzzy fixed point results for fuzzy mappings under generalized almost $\mathbf{F}$-contractions in the context of a controlled metric space, which generalize many preexisting results in the literature. As an application, we establish some multivalued fixed point results. For justification of our results, we provide a nontrivial example.
\end{abstract}

MSC: 46T99; 47H10; $54 \mathrm{H} 25$

Keywords: $\alpha$-fuzzy fixed points; Generalized almost $\mathbb{F}$-contraction; Controlled metric space

\section{Introduction}

The Banach fixed point theorem (BFPT) [1] is an important tool in fixed point theory. It guarantees the existence and uniqueness of a fixed point of certain self-mappings on metric spaces. It has various applications in several branches of mathematics. There are many extensions and generalizations of the BFPT in the literature; see [2-7]. Berinde [8,9] studied various contractive-type mappings and introduced the concept of almost contractions.

Definition 1.1 ([8]) A mapping $T: \mathcal{W} \rightarrow \mathcal{W}$ on a metric space $(\mathcal{W}, d)$ is called an almost contraction if there exist $0 \leq \lambda<1$ and $€ \geq 0$ such that

$$
d\left(T \omega_{1}, T \omega_{2}\right) \leq \lambda d\left(\omega_{1}, \omega_{2}\right)+\succeq d\left(\omega_{2}, T \omega_{1}\right)
$$

for all $\omega_{1}, \omega_{2} \in \mathcal{W}$.

Further, Berinde [9] generalized Definition 1.1 in the following way.

(c) The Author(s) 2021. This article is licensed under a Creative Commons Attribution 4.0 International License, which permits use, sharing, adaptation, distribution and reproduction in any medium or format, as long as you give appropriate credit to the original author(s) and the source, provide a link to the Creative Commons licence, and indicate if changes were made. The images or other third party material in this article are included in the article's Creative Commons licence, unless indicated otherwise in a credit line to the material. If material is not included in the article's Creative Commons licence and your intended use is not permitted by statutory regulation or exceeds the permitted use, you will need to obtain permission directly from the copyright holder. To view a copy of this licence, visit http://creativecommons.org/licenses/by/4.0/. 
Definition 1.2 ([9]) A mapping $T: \mathcal{W} \rightarrow \mathcal{W}$ on a metric space $(\mathcal{W}, d)$ is called a generalized almost contraction if there exist $0 \leq \lambda<1$ and $€ \geq 0$ such that

$$
\begin{aligned}
\left.d\left(T \omega_{1}, T \omega_{2}\right)\right) \leq & \lambda d\left(\omega_{1}, \omega_{2}\right) \\
& +\succeq \min \left\{d\left(\omega_{1}, T\left(\omega_{1}\right)\right), d\left(\omega_{2}, T\left(\omega_{2}\right)\right), d\left(\omega_{1}, T\left(\omega_{2}\right)\right), d\left(\omega_{2}, T\left(\omega_{1}\right)\right)\right\}
\end{aligned}
$$

for all $\omega_{1}, \omega_{2} \in \mathcal{W}$.

Wardowski [10] introduced a new type of contractions, called F-contractions, and established a related fixed point theorem in the context of complete metric spaces.

Definition 1.3 ([10]) A mapping $T: \mathcal{W} \rightarrow \mathcal{W}$ on a metric space $(\mathcal{W}, d)$ is called an Fcontraction if there exists $\Omega>0$ such that

$$
d\left(T \omega_{1}, T \omega_{2}\right)>0 \quad \Longrightarrow \quad \Omega+\mathbf{F}\left(d\left(T \omega_{1}, T \omega_{2}\right)\right) \leq \mathbf{F}\left(d\left(\omega_{1}, \omega_{2}\right)\right)
$$

for all $\omega_{1}, \omega_{2} \in \mathcal{W}$, where $\mathbf{F}:(0, \infty) \rightarrow \mathbb{R}$ is a function satisfying the following axioms:

(C1) $\mathbf{F}$ is strictly nondecreasing;

(C2) for each sequence $\left\{a_{n}\right\} \subset(0, \infty)$ of positive real numbers, $\lim _{n \rightarrow \infty} a_{n}=0$ if and only if $\lim _{n \rightarrow \infty} \mathbf{F}\left(a_{n}\right)=-\infty$;

(C3) for each sequence $\left\{a_{n}\right\} \subset(0, \infty)$ such that $\lim _{n \rightarrow \infty} a_{n}=0$, there exists $l \in(0,1)$ such that $\lim _{n \rightarrow \infty}\left(a_{n}\right)^{l} \mathbf{F}\left(a_{n}\right)=0$.

The following works deal with $F$-contractions: [11-16]. Afterward, Altun et al. [17] modified Definition 1.3 by adding the following condition:

(C4) $\mathbf{F}(\inf \mathbf{A})=\inf \mathbf{F}(\mathbf{A})$ for all $\mathbf{A} \subset(0, \infty)$ with $\inf \mathbf{A}>0$.

We denote by $\mathcal{F}$ the family of all functions $\mathbf{F}$ satisfying $(\mathrm{C} 1)-(\mathrm{C} 4)$.

Nadler [18] derived the multivalued version of Banach fixed point theorem by using the Hausdorff metric over the family of nonempty closed bounded subsets of a complete metric space. We denote by $C L B(\mathcal{W})$ the family of nonempty closed bounded subsets and by $C L D(\mathcal{W})$ the family of nonempty closed subsets of $\mathcal{W}$. Recently, Kamran et al. [19] introduced the concept of an extended $b$-metric space, which generalized the notion of a $b$-metric space $[20,21]$ by replacing the constant with a function depending on two variables.

Definition 1.4 ([19]) Let $\mathcal{W}$ be a nonempty set, and let $\sigma: \mathcal{W} \times \mathcal{W} \rightarrow[1, \infty)$. Then a function $d_{\sigma}: \mathcal{W} \times \mathcal{W} \rightarrow[0, \infty)$ is called an extended $b$-metric if for all $\omega_{1}, \omega_{2}, \omega_{3} \in \mathcal{W}$, it satisfies the following axioms:

(i) $d_{\sigma}\left(\omega_{1}, \omega_{2}\right)=0$ iff $\omega_{1}=\omega_{2}$,

(ii) $d_{\sigma}\left(\omega_{1}, \omega_{2}\right)=d_{\sigma}\left(\omega_{2}, \omega_{1}\right)$,

(iii) $d_{\sigma}\left(\omega_{1}, \omega_{3}\right) \leq \sigma\left(\omega_{1}, \omega_{3}\right)\left[d_{\sigma}\left(\omega_{1}, \omega_{2}\right)+d_{\sigma}\left(\omega_{2}, \omega_{3}\right)\right]$.

The pair $\left(\mathcal{W}, d_{\sigma}\right)$ is called an extended $b$-metric space.

Later on, several researchers worked on fixed point results in the context of extended $b$-metric spaces; see [22-25]. In the same direction, Mlaiki et al. [26] gave the idea of a controlled-type metric space (for further extensions, see [27]), which generalizes the notion of a $b$-metric space. 
Definition 1.5 ([26]) Let $\mathcal{W}$ be a nonempty set, and let $\sigma: \mathcal{W} \times \mathcal{W} \rightarrow[1, \infty)$. Then a function $d_{\sigma}: \mathcal{W} \times \mathcal{W} \rightarrow[0, \infty)$ is called a controlled metric if for all $\omega_{1}, \omega_{2}, \omega_{3} \in \mathcal{W}$, it satisfies the following axioms:

(i) $d_{\sigma}\left(\omega_{1}, \omega_{2}\right)=0$ iff $\omega_{1}=\omega_{2}$,

(ii) $d_{\sigma}\left(\omega_{1}, \omega_{2}\right)=d_{\sigma}\left(\omega_{2}, \omega_{1}\right)$,

(iii) $d_{\sigma}\left(\omega_{1}, \omega_{3}\right) \leq \sigma\left(\omega_{1}, \omega_{2}\right) d_{\sigma}\left(\omega_{1}, \omega_{2}\right)+\sigma\left(\omega_{2}, \omega_{3}\right) d_{\sigma}\left(\omega_{2}, \omega_{3}\right)$.

The pair $\left(\mathcal{W}, d_{\sigma}\right)$ is called a controlled metric space.

Remark 1.1 Every controlled metric space is a generalization of a $b$-metric space and is different from an extended $b$-metric space.

Example 1.1 Let $\mathcal{W}=[0, \infty)$. Define $d_{\sigma}: \mathcal{W} \times \mathcal{W} \rightarrow[0, \infty)$ as

$$
d_{\sigma}\left(\omega_{1}, \omega_{2}\right)= \begin{cases}0 & \text { if } \omega_{1}=\omega_{2} \\ \frac{1}{\omega_{1}} & \text { if } \omega_{1} \geq 1 \text { and } \omega_{2} \in[0,1) \\ \frac{1}{\omega_{2}} & \text { if } \omega_{2} \geq 1 \text { and } \omega_{1} \in[0,1) \\ 1 & \text { otherwise }\end{cases}
$$

Hence $\left(\mathcal{W}, d_{\sigma}\right)$ is a controlled metric space, where $\sigma: \mathcal{W} \times \mathcal{W} \rightarrow[1, \infty)$ is defined by

$$
\sigma\left(\omega_{1}, \omega_{2}\right)= \begin{cases}1 & \text { if } \omega_{1}, \omega_{2} \in[0,1) \\ \max \left\{\omega_{1}, \omega_{2}\right\} & \text { otherwise }\end{cases}
$$

For other definitions and information on the topology induced by $d_{\sigma}$, see [26]. In [28], Alamgir et al. established a Pompieu-Hausdorff metric over the family of nonempty closed subsets of a controlled metric space $W$ as follows.

Definition 1.6 ([28]) Let A, B be nonempty closed subsets of a controlled metric space $\left(\mathcal{W}, d_{\sigma}\right)$. Define $H_{\sigma}: C L D(\mathcal{W}) \times C L D(\mathcal{W}) \rightarrow[0, \infty]$ by

$$
H_{\sigma}(\mathbf{A}, \mathbf{B})= \begin{cases}\max \left\{\sup _{a \in \mathbf{A}} d_{\sigma}(a, \mathbf{B}), \sup _{b \in \mathbf{B}} d_{\sigma}(b, \mathbf{A})\right\} & \text { if the maximum exists; } \\ \infty & \text { otherwise. }\end{cases}
$$

Theorem 1.1 ([28]) Let $\left(\mathcal{W}, d_{\sigma}\right)$ be a controlled metric space. Then the mapping $H_{\sigma}$ : $C L D(\mathcal{W}) \times C L D(\mathcal{W}) \rightarrow[0, \infty]$ is a Pompieu-Hausdorff controlled metric on $C L D(\mathcal{W})$.

On the other hand, in 1981, Heilpern [29] used fuzzy sets [30] to introduce a class of fuzzy mappings, which is a generalization of multivalued mappings and proved a fixed point theorem for fuzzy contraction mappings in metric spaces. The result introduced by Heilpern is a fuzzy generalization of the Banach fixed point theorem. Consequently, several authors studied and generalized fuzzy fixed point theorems in many directions; see [31-38]. In this paper, we prove some common $\alpha$-fuzzy fixed point results for fuzzy mappings under generalized almost F-contractions in the context of controlled metric spaces, which generalize many preexisting results in the literature. At the end, we give an example for the justification of our main result. 


\section{Main results}

In this section, we define fuzzy sets, fuzzy mappings, and $\alpha$-fuzzy fixed points and prove some common $\alpha$ fuzzy fixed point results in the context of controlled metric spaces.

Definition 2.1 Let $\left(\mathcal{W}, d_{\sigma}\right)$ be a controlled metric space with $\sigma: \mathcal{W} \times \mathcal{W} \rightarrow[1, \infty)$. Then a fuzzy set $\mathbf{A}_{\sigma}$ in $\mathcal{W}$ is characterized by a membership function

$$
\mathbb{F}_{\mathbf{A}_{\sigma}}: \mathcal{W} \rightarrow[0,1]
$$

which assigns to every member of $\mathcal{W}$ a membership grade in $\mathbf{A}_{\sigma}$.

We denote by $\mathbb{F}_{\sigma}(\mathcal{W})$ the collection of all fuzzy sets in $\mathcal{W}$. Let $\mathbf{A}_{\sigma} \in \mathbb{F}_{\sigma}(\mathcal{W})$ and $\alpha \in[0,1]$. Then the $\alpha$-level set of $\mathbf{A}_{\sigma}$ is denoted by $\left[\mathbf{A}_{\sigma}\right]_{\alpha}$ and is defined as

$$
\begin{aligned}
& {\left[\mathbf{A}_{\sigma}\right]_{\alpha}=\left\{\mu \in \mathcal{W}: \mathbf{A}_{\sigma}(\mu) \geq \alpha\right\}, \quad \alpha \in(0,1],} \\
& {\left[\mathbf{A}_{\sigma}\right]_{0}=\overline{\left\{\mu \in \mathcal{W}: \mathbf{A}_{\sigma}(\mu)>0\right\}},}
\end{aligned}
$$

where $\overline{\mathbf{B}}$ denotes the closure of $\mathbf{B}$. Clearly, $\left[\mathbf{A}_{\sigma}\right]_{\alpha}$ and $\left[\mathbf{A}_{\sigma}\right]_{0}$ are subsets of the controlled metric space $\mathcal{W}$. For $\mathbf{A}_{\sigma}, \mathbf{B}_{\sigma} \in \mathbf{F}_{\sigma}(\mathcal{W})$, a fuzzy set $\mathbf{A}_{\sigma}$ is said to be more accurate than a fuzzy set $\mathbf{B}_{\sigma}$, denoted by $\mathbf{A}_{\sigma} \subset \mathbf{B}_{\sigma}$, if $f_{\mathbf{A}_{\sigma}}(\mu) \leq f_{\mathbf{B}_{\sigma}}(\mu)$ for each $\mu \in \mathcal{W}$. Now, for $\mu \in \mathcal{W}$, $\mathbf{A}_{\sigma}, \mathbf{B}_{\sigma} \in \mathbb{F}_{\sigma}(\mathcal{W}), \alpha \in[0,1]$, and $\left[\mathbf{A}_{\sigma}\right]_{\alpha},\left[\mathbf{B}_{\sigma}\right]_{\alpha} \in C L B(\mathcal{W})$, define

$$
\begin{aligned}
& \rho_{\alpha}\left(\mu,\left[\mathbf{A}_{\sigma}\right]_{\alpha}\right)=\inf \left\{d(\mu, a): a \in\left[\mathbf{A}_{\sigma}\right]_{\alpha}\right\}, \\
& \rho_{\alpha}\left(\left[\mathbf{A}_{\sigma}\right]_{\alpha},\left[\mathbf{B}_{\sigma}\right]_{\alpha}\right)=\inf \left\{d(a, b): a \in\left[\mathbf{A}_{\sigma}\right]_{\alpha}, b \in\left[\mathbf{B}_{\sigma}\right]_{\alpha}\right\}, \\
& \rho\left(\left[\mathbf{A}_{\sigma}\right]_{\alpha},\left[\mathbf{B}_{\sigma}\right]_{\alpha}\right)=\sup _{\alpha} \rho_{\alpha}\left(\left[\mathbf{A}_{\sigma}\right]_{\alpha},\left[\mathbf{B}_{\sigma}\right]_{\alpha}\right) .
\end{aligned}
$$

Remark 2.1 By Theorem 1.1 the function $H_{\sigma}: C L B(\mathcal{W}) \times C L B(\mathcal{W}) \rightarrow[0, \infty]$ defined by

$$
\begin{aligned}
& H_{\sigma}\left(\left[\mathbf{A}_{\sigma}\right]_{\alpha},\left[\mathbf{B}_{\sigma}\right]_{\alpha}\right) \\
& \quad= \begin{cases}\max \left\{\sup _{a \in\left[\mathbf{A}_{\sigma}\right]_{\alpha}} d\left(a,\left[\mathbf{B}_{\sigma}\right]_{\alpha}\right), \sup _{b \in\left[\mathbf{B}_{\sigma}\right]_{\alpha}} d\left(b,\left[\mathbf{A}_{\sigma}\right]_{\alpha}\right)\right\} & \text { if the maximum exists, } \\
\infty & \text { otherwise, }\end{cases}
\end{aligned}
$$

is a generalized Hausdorff controlled fuzzy metric on $C L B(\mathcal{W})$.

Definition 2.2 Let $\mathbf{S}, \mathbf{T}$ be fuzzy mappings from $\mathcal{W}$ into $\Gamma(\mathcal{W})$. Then

(i) An element $\mu \in \mathcal{W}$ is called an $\alpha$-fuzzy fixed point of $\mathbf{T}$ if there exists $\alpha_{\mathbf{T}}(\mu) \in(0,1]$ such that $\mu \in[\mathbf{T} \mu]_{\alpha_{\mathbf{T}}(\mu)}$.

(ii) An element $\mu \in \mathcal{W}$ is called a common $\alpha$-fuzzy fixed point of $\mathbf{S}$ and $\mathbf{T}$ if there exist $\alpha_{\mathbf{S}}(\mu), \alpha_{\mathbf{T}}(\mu) \in(0,1]$ such that $\mu \in[\mathbf{S} \mu]_{\alpha_{\mathbf{S}}(\mu)} \cap[\mathbf{T} \mu]_{\alpha_{\mathbf{T}}(\mu)}$.

(iii) For $\alpha=1, \mu$ is called a common fixed point of fuzzy mappings.

Lemma 2.1 Let $\left(\mathcal{W}, d_{\sigma}\right)$ be a controlled metric space, and let $\mathbf{A}, \mathbf{B} \in C L B(\mathcal{W})$. Then for each $a \in \mathbf{A}$,

$$
d_{\sigma}(a, \mathbf{B}) \leq H_{\sigma}(\mathbf{A}, \mathbf{B}) .
$$


Proof Let us suppose on the contrary that for each $a \in \mathbf{A}$,

$$
d_{\sigma}(a, \mathbf{B})>H_{\sigma}(\mathbf{A}, \mathbf{B}) .
$$

From Definition 1.6 we have that for each $a \in \mathbf{A}$,

$$
d_{\sigma}(a, \mathbf{B}) \leq H_{\sigma}(\mathbf{A}, \mathbf{B}) .
$$

Hence from equations (4) and (5) we get

$$
H_{\sigma}(\mathbf{A}, \mathbf{B})<d_{\sigma}(a, \mathbf{B}) \leq H_{\sigma}(\mathbf{A}, \mathbf{B}),
$$

a contradiction.

Theorem 2.1 Let $\left(\mathcal{W}, d_{\sigma}\right)$ be a complete controlled metric space, and let $\mathbf{S}, \mathbf{T}$ be fuzzy mappings from $\mathcal{W}$ into $\Gamma(\mathcal{W})$. Suppose for each $\omega_{1} \in \mathcal{W}$, there exist $\alpha_{\mathbf{S}}\left(\omega_{1}\right), \alpha_{\mathbf{T}}\left(\omega_{2}\right) \in(0,1]$ such that $\left[\mathbf{S} \omega_{1}\right]_{\alpha_{\mathbf{S}}\left(\omega_{1}\right)},\left[\mathbf{T} \omega_{2}\right]_{\alpha_{\mathbf{T}}\left(\omega_{2}\right)}$ are nonempty closed subsets of $\mathcal{W}$. Suppose that there exist some $\mathbf{F} \in \mathcal{F}, \Omega>0$, and $€ \geq 0$ such that

$$
\Omega+\mathbf{F}\left(H_{\sigma}\left(\left[\mathbf{S} \omega_{1}\right]_{\alpha_{\mathbf{S}}\left(\omega_{1}\right)},\left[\mathbf{T} \omega_{2}\right]_{\alpha_{\mathbf{T}}\left(\omega_{2}\right)}\right) \leq \mathbf{F}\left(d_{\sigma}\left(\omega_{1}, \omega_{2}\right)\right)+\succeq\left(M\left(\omega_{1}, \omega_{2}\right)\right)\right.
$$

for all $\omega_{1}, \omega_{2} \in \mathcal{W}$ with $H_{\sigma}\left(\left[\mathbf{S} \omega_{1}\right]_{\alpha_{\mathbf{S}}\left(\omega_{1}\right)},\left[\mathbf{T} \omega_{2}\right]_{\alpha_{\mathbf{T}}\left(\omega_{2}\right)}\right)>0$, where

$$
\begin{aligned}
M\left(\omega_{1}, \omega_{2}\right)= & \min \left\{d_{\sigma}\left(\omega_{1},\left[\mathbf{S} \omega_{1}\right]_{\alpha_{\mathbf{S}}\left(\omega_{1}\right)}\right), d_{\sigma}\left(\omega_{2},\left[\mathbf{T} \omega_{2}\right]_{\alpha_{\mathbf{T}}\left(\omega_{2}\right)}\right),\right. \\
& \left.d_{\sigma}\left(\omega_{1},\left[\mathbf{T} \omega_{2}\right]_{\alpha_{\mathbf{T}}\left(\omega_{2}\right)}\right), d_{\sigma}\left(\omega_{2},\left[\mathbf{S} \omega_{1}\right]_{\alpha_{\mathbf{S}}\left(\omega_{1}\right)}\right)\right\}
\end{aligned}
$$

Then there exists a common $\alpha$-fuzzy fixed point of $\mathbf{S}$ and $\mathbf{T}$.

Proof Let us take an arbitrary $\omega_{0} \in \mathcal{W}$. Then by the hypothesis there exists $\alpha_{\mathbf{S}}\left(\omega_{0}\right) \in(0,1]$ such that $\left[\mathbf{S} \omega_{0}\right]_{\alpha_{\mathbf{S}}\left(\omega_{0}\right)}$ is a nonempty closed subset of $\mathcal{W}$. Let $\omega_{1} \in\left[\mathbf{S} \omega_{0}\right]_{\alpha_{\mathbf{S}}\left(\omega_{0}\right)}$. For such $\omega_{1}$, there exists $\alpha_{\mathbf{T}}\left(\omega_{1}\right) \in(0,1]$ such that $\left[\mathbf{T} \omega_{1}\right]_{\alpha_{\mathbf{T}}\left(\omega_{1}\right)}$ is a nonempty closed subset of $\mathcal{W}$. From Lemma 2.1, condition (C1) of Definition 1.3, and (6) we can write

$$
\begin{aligned}
\Omega+\mathbf{F}\left(d_{\sigma}\left(\omega_{1},\left[\mathbf{T} \omega_{1}\right]_{\alpha_{\mathbf{T}}\left(\omega_{1}\right)}\right)\right. & \leq \Omega+\mathbf{F}\left(H_{\sigma}\left(\left[\mathbf{S} \omega_{0}\right]_{\alpha_{\mathbf{S}}\left(\omega_{0}\right)},\left[\mathbf{T} \omega_{1}\right]_{\alpha_{\mathbf{T}}\left(\omega_{1}\right)}\right)\right. \\
& \leq \mathbf{F}\left(d_{\sigma}\left(\omega_{0}, \omega_{1}\right)\right)+\succeq\left(M\left(\omega_{0}, \omega_{1}\right)\right),
\end{aligned}
$$

where

$$
\begin{aligned}
M\left(\omega_{0}, \omega_{1}\right)= & \min \left\{d_{\sigma}\left(\omega_{0},\left[\mathbf{S} \omega_{0}\right]_{\alpha_{\mathbf{S}}\left(\omega_{0}\right)}\right), d_{\sigma}\left(\omega_{1},\left[\mathbf{T} \omega_{1}\right]_{\alpha_{\mathbf{T}}\left(\omega_{1}\right)}\right),\right. \\
& \left.d_{\sigma}\left(\omega_{0},\left[\mathbf{T} \omega_{1}\right]_{\alpha_{\mathbf{T}}\left(\omega_{1}\right)}\right), d_{\sigma}\left(\omega_{1},\left[\mathbf{S} \omega_{0}\right]_{\alpha_{\mathbf{S}}\left(\omega_{0}\right)}\right)\right\}
\end{aligned}
$$

From condition $(C 4)$ we can write

$$
\mathbf{F}\left(d_{\sigma}\left(\omega_{1},\left[\mathbf{T} \omega_{1}\right]_{\alpha_{\mathbf{T}}\left(\omega_{1}\right)}\right)=\inf _{y \in\left[\mathbf{T} \omega_{1}\right]_{\alpha}\left(\omega_{1}\right)} \mathbf{F}\left(d_{\sigma}\left(\omega_{1}, y\right)\right) .\right.
$$


Thus we have

$$
\begin{aligned}
\Omega+ & \inf _{y \in\left[\mathbf{T} \omega_{1}\right]_{\mathbf{T}_{\mathbf{T}}\left(\omega_{1}\right)}} \mathbf{F}\left(d_{\sigma}\left(\omega_{1}, y\right)\right. \\
\leq & \mathbf{F}\left(d_{\sigma}\left(\omega_{0}, \omega_{1}\right)\right)+\succeq \min \left\{d_{\sigma}\left(\omega_{0},\left[\mathbf{S} \omega_{0}\right]_{\alpha_{\mathbf{S}}\left(\omega_{0}\right)}\right), d_{\sigma}\left(\omega_{1},\left[\mathbf{T} \omega_{1}\right]_{\alpha_{\mathbf{T}}\left(\omega_{1}\right)}\right),\right. \\
& \left.d_{\sigma}\left(\omega_{0},\left[\mathbf{T} \omega_{1}\right]_{\alpha_{\mathbf{T}}\left(\omega_{1}\right)}\right), d_{\sigma}\left(\omega_{1},\left[\mathbf{S} \omega_{0}\right]_{\alpha_{\mathbf{S}}\left(\omega_{0}\right)}\right)\right\} .
\end{aligned}
$$

Then there exists $\omega_{2} \in\left[\mathbf{T} \omega_{1}\right]_{\alpha_{\mathbf{T}}\left(\omega_{1}\right)}$ such that

$$
\begin{aligned}
\Omega & +\mathbf{F}\left(d_{\sigma}\left(\omega_{1}, \omega_{2}\right)\right. \\
& \leq \mathbf{F}\left(d_{\sigma}\left(\omega_{0}, \omega_{1}\right)\right)+七 \min \left\{d_{\sigma}\left(\omega_{0}, \omega_{1}\right), d_{\sigma}\left(\omega_{1}, \omega_{2}\right), d_{\sigma}\left(\omega_{0}, \omega_{2}\right), d_{\sigma}\left(\omega_{1}, \omega_{1}\right)\right\} \\
& =\mathbf{F}\left(d_{\sigma}\left(\omega_{0}, \omega_{1}\right)\right) .
\end{aligned}
$$

For this $\omega_{2}$, there exists $\alpha_{\mathbf{S}}\left(\omega_{2}\right) \in(0,1]$ such that $\left[\mathbf{S} \omega_{2}\right]_{\alpha_{\mathbf{S}}\left(\omega_{2}\right)}$ is a nonempty closed subset of $\mathcal{W}$. From Lemma 2.1, condition (C1) of Definition 1.3, and (6) we have

$$
\begin{aligned}
\Omega+\mathbf{F}\left(d_{\sigma}\left(\omega_{2},\left[\mathbf{S} \omega_{2}\right]_{\alpha_{\mathbf{S}}\left(\omega_{2}\right)}\right)\right. & \leq \Omega+\mathbf{F}\left(H_{\sigma}\left(\left[\mathbf{T} \omega_{1}\right]_{\alpha_{\mathbf{T}}\left(\omega_{1}\right)},\left[\mathbf{S} \omega_{2}\right]_{\alpha_{\mathbf{S}}\left(\omega_{2}\right)}\right)\right. \\
& \leq \Omega+\mathbf{F}\left(H_{\sigma}\left(\left[\mathbf{S} \omega_{2}\right]_{\alpha_{\mathbf{S}}\left(\omega_{2}\right)},\left[\mathbf{T} \omega_{1}\right]_{\alpha_{\mathbf{T}}\left(\omega_{1}\right)}\right)\right. \\
& \leq \mathbf{F}\left(d_{\sigma}\left(\omega_{2}, \omega_{1}\right)\right)+\mathrm{\longleftarrow}\left(M\left(\omega_{2}, \omega_{1}\right)\right),
\end{aligned}
$$

where

$$
\begin{aligned}
M\left(\omega_{2}, \omega_{1}\right)= & \min \left\{d_{\sigma}\left(\omega_{2},\left[\mathbf{S} \omega_{2}\right]_{\alpha_{\mathbf{S}}\left(\omega_{2}\right)}\right), d_{\sigma}\left(\omega_{1},\left[\mathbf{T} \omega_{1}\right]_{\alpha_{\mathbf{T}}\left(\omega_{1}\right)}\right),\right. \\
& \left.d_{\sigma}\left(\omega_{2},\left[\mathbf{T} \omega_{1}\right]_{\alpha_{\mathbf{T}}\left(\omega_{1}\right)}\right), d_{\sigma}\left(\omega_{1},\left[\mathbf{S} \omega_{2}\right]_{\alpha_{\mathbf{S}}\left(\omega_{2}\right)}\right)\right\} .
\end{aligned}
$$

From condition (C4), we can write

$$
\mathbf{F}\left(d_{\sigma}\left(\omega_{2},\left[\mathbf{S} \omega_{2}\right]_{\alpha_{\mathbf{S}}}\left(\omega_{2}\right)\right)\right)=\inf _{\left.y^{\prime} \in\left[\mathbf{S} \omega_{2}\right]_{\alpha} \mathbf{S}^{(} \omega_{2}\right)} \mathbf{F}\left(d_{\sigma}\left(\omega_{2}, y^{\prime}\right)\right) .
$$

Then we have

$$
\begin{aligned}
\Omega+ & \inf _{y^{\prime} \in\left[\mathbf{S} \omega_{2}\right]_{\alpha_{\mathbf{S}}\left(\omega_{2}\right)}} \mathbf{F}\left(d_{\sigma}\left(\omega_{2}, y^{\prime}\right)\right) \\
\leq & \mathbf{F}\left(d_{\sigma}\left(\omega_{2}, \omega_{1}\right)\right)+七 \min \left\{d_{\sigma}\left(\omega_{2},\left[\mathbf{S} \omega_{2}\right]_{\alpha_{\mathbf{S}}\left(\omega_{2}\right)}\right), d_{\sigma}\left(\omega_{1},\left[\mathbf{T} \omega_{1}\right]_{\alpha_{\mathbf{T}}\left(\omega_{1}\right)}\right),\right. \\
& \left.d_{\sigma}\left(\omega_{2},\left[\mathbf{T} \omega_{1}\right]_{\alpha_{\mathbf{T}}\left(\omega_{1}\right)}\right), d_{\sigma}\left(\omega_{1},\left[\mathbf{S} \omega_{2}\right]_{\alpha_{\mathbf{S}}\left(\omega_{2}\right)}\right)\right\} .
\end{aligned}
$$

Thus there exists $\omega_{3} \in\left[\mathbf{S} \omega_{2}\right]_{\alpha_{\mathbf{S}}\left(\omega_{2}\right)}$ such that

$$
\begin{aligned}
\Omega & +\mathbf{F}\left(d_{\sigma}\left(\omega_{2}, \omega_{3}\right)\right) \\
& \leq \mathbf{F}\left(d_{\sigma}\left(\omega_{1}, \omega_{2}\right)\right)+\succeq \min \left\{d_{\sigma}\left(\omega_{2}, \omega_{3}\right), d_{\sigma}\left(\omega_{1}, \omega_{2}\right), d_{\sigma}\left(\omega_{2}, \omega_{2}\right), d_{\sigma}\left(\omega_{1}, \omega_{3}\right)\right\} .
\end{aligned}
$$

This implies that

$$
\Omega+\mathbf{F}\left(d_{\sigma}\left(\omega_{2}, \omega_{3}\right)\right) \leq \mathbf{F}\left(d_{\sigma}\left(\omega_{1}, \omega_{2}\right)\right)
$$


By continuing the same procedure recursively we obtain a sequence $\left\{\omega_{n}\right\}_{n=0}^{\infty}$ in $\mathcal{W}$ such that $\omega_{2 n+1} \in\left[\mathbf{S} \omega_{2 n}\right]_{\alpha_{\mathbf{S}}\left(\omega_{2 n}\right)}, \omega_{2 n+2} \in\left[\mathbf{T} \omega_{2 n+1}\right]_{\alpha_{\mathbf{T}}\left(\omega_{2 n+1}\right)}$. Also,

$$
\Omega+\mathbf{F}\left(d_{\sigma}\left(\omega_{2 n+1}, \omega_{2 n+2}\right)\right) \leq \mathbf{F}\left(d_{\sigma}\left(\omega_{2 n}, \omega_{2 n+1}\right)\right)
$$

and

$$
\Omega+\mathbf{F}\left(d_{\sigma}\left(\omega_{2 n+2}, \omega_{2 n+3}\right)\right) \leq \mathbf{F}\left(d_{\sigma}\left(\omega_{2 n+1}, \omega_{2 n+2}\right)\right)
$$

for all $n \in \mathbb{N}$. From equations (8) and (9) we have

$$
\Omega+\mathbf{F}\left(d_{\sigma}\left(\omega_{n}, \omega_{n+1}\right)\right) \leq \mathbf{F}\left(d_{\sigma}\left(\omega_{n-1}, \omega_{n}\right)\right)
$$

Therefore

$$
\begin{aligned}
\mathbf{F}\left(d_{\sigma}\left(\omega_{n}, \omega_{n+1}\right)\right) & \leq \mathbf{F}\left(d_{\sigma}\left(\omega_{n-1}, \omega_{n}\right)\right)-\Omega \leq \mathbf{F}\left(d_{\sigma}\left(\omega_{n-2}, \omega_{n-1}\right)\right)-2 \Omega \leq \cdots \\
& \leq \mathbf{F}\left(d_{\sigma}\left(\omega_{0}, \omega_{1}\right)\right)-n \Omega
\end{aligned}
$$

By taking the limit as $n \rightarrow \infty$ in equation (10) we get $\lim _{n \rightarrow \infty} \mathbb{F}\left(d_{\sigma}\left(\omega_{n}, \omega_{n+1}\right)\right)=-\infty$. Next, from condition $(C 2)$ of Definition 1.3 we have

$$
\lim _{n \rightarrow \infty} d_{\sigma}\left(\omega_{n}, \omega_{n+1}\right)=0
$$

Also, by condition $(C 3)$ of Definition 1.3 there exists $l \in(0,1)$ such that

$$
\lim _{n \rightarrow \infty}\left(d_{\sigma}\left(\omega_{n}, \omega_{n+1}\right)\right)^{l} \mathbf{F}\left(d_{\sigma}\left(\omega_{n}, \omega_{n+1}\right)\right)=0
$$

From equation (10) we have that for all $n \in \mathbb{N}$,

$$
\begin{aligned}
& \left(d_{\sigma}\left(\omega_{n}, \omega_{n+1}\right)\right)^{l} \mathbf{F}\left(d_{\sigma}\left(\omega_{n}, \omega_{n+1}\right)\right)-\left(d_{\sigma}\left(\omega_{n}, \omega_{n+1}\right)\right)^{l} \mathbf{F}\left(d_{\sigma}\left(\omega_{0}, \omega_{1}\right)\right) \\
& \quad \leq-\left(d_{\sigma}\left(\omega_{n}, \omega_{n+1}\right)\right)^{l} n \Omega \leq 0 .
\end{aligned}
$$

By letting $n \rightarrow \infty$ in (11) we obtain

$$
\lim _{n \rightarrow \infty} n\left(d_{\sigma}\left(\omega_{n}, \omega_{n+1}\right)\right)^{l}=0
$$

By equation (12) there exists $n_{1} \in \mathbb{N}$ such that $n\left(\mathbf{F}\left(d_{\sigma}\left(\omega_{n}, \omega_{n+1}\right)\right)\right)^{l} \leq 1$ for all $n \geq n_{1}$. Thus, for all $n \geq n_{1}$, we have

$$
d_{\sigma}\left(\omega_{n}, \omega_{n+1}\right) \leq \frac{1}{n^{\frac{1}{l}}}
$$

From the triangle inequality and equation (13) for $m>n \geq n_{1}$, we have

$$
\begin{aligned}
d_{\sigma}\left(\omega_{n}, \omega_{m}\right) & \leq \sigma\left(\omega_{n}, \omega_{n+1}\right) d_{\sigma}\left(\omega_{n}, \omega_{n+1}\right)+\sigma\left(\omega_{n+1}, \omega_{m}\right) d_{\sigma}\left(\omega_{n+1}, \omega_{m}\right) \\
& \leq \sigma\left(\omega_{n}, \omega_{n+1}\right) d_{\sigma}\left(\omega_{n}, \omega_{n+1}\right)+\sigma\left(\omega_{n}, \omega_{m}\right) \sigma\left(\omega_{n+1}, \omega_{n+2}\right) d_{\sigma}\left(\omega_{n+1}, \omega_{n+2}\right)
\end{aligned}
$$




$$
\begin{aligned}
& \quad+\sigma\left(\omega_{n}, \omega_{m}\right) \sigma\left(\omega_{n+2}, \omega_{m}\right) d_{\sigma}\left(\omega_{n+2}, \omega_{m}\right) \\
& \vdots \\
& \leq \\
& \leq\left(\omega_{n}, \omega_{n+1}\right) d_{\sigma}\left(\omega_{n}, \omega_{n+1}\right)+\sum_{i=1}^{m-2}\left(\prod_{j=1}^{i} \sigma\left(\omega_{j}, \omega_{m}\right)\right) \sigma\left(\omega_{i}, \omega_{i+1}\right) d_{\sigma}\left(\omega_{i}, \omega_{i+1}\right) \\
& \quad+\prod_{j=1}^{m-1} \sigma\left(\omega_{j}, \omega_{m}\right) \sigma\left(\omega_{m-1}, \omega_{m}\right) d_{\sigma}\left(\omega_{m-1}, \omega_{m}\right) \\
& \leq \sigma\left(\omega_{n}, \omega_{n+1}\right) d_{\sigma}\left(\omega_{n}, \omega_{n+1}\right)+\sum_{i=1}^{m-1}\left(\prod_{j=1}^{i} \sigma\left(\omega_{j}, \omega_{m}\right)\right) \sigma\left(\omega_{i}, \omega_{i+1}\right) d_{\sigma}\left(\omega_{i}, \omega_{i+1}\right) \\
& \leq \sigma\left(\omega_{n}, \omega_{n+1}\right) \frac{1}{n^{\frac{1}{\tau}}}+\sum_{i=1}^{\infty}\left(\prod_{j=1}^{i} \sigma\left(\omega_{j}, \omega_{m}\right)\right) \sigma\left(\omega_{i}, \omega_{i+1}\right) \frac{1}{i^{\frac{1}{\tau}}}
\end{aligned}
$$

Since $\lim _{n, m \rightarrow \infty} \sigma\left(\omega_{n+1}, \omega_{m}\right) l<1$ for all $\omega_{n}, \omega_{m} \in \mathcal{W}$, the series $\sum_{i=1}^{\infty}\left(\prod_{j=1}^{i} \sigma\left(\omega_{j}, \omega_{m}\right)\right) \sigma\left(\omega_{i}\right.$, $\left.\omega_{i+1}\right) \frac{1}{i^{\frac{1}{l}}}$ converges by the ratio test for each $m \in \mathbb{N}$. Therefore, by taking the limit as $n \rightarrow \infty$ in the above inequality we get $d_{\sigma}\left(\omega_{n}, \omega_{m}\right) \rightarrow 0$. Since $\mathcal{W}$ is complete, there exists $\rho \in \mathcal{W}$ such that $\lim _{n \rightarrow \infty} \omega_{n}=\rho$. Next, we prove that $\rho$ is a fixed point of T. Suppose on the contrary that $\rho$ is not a fixed point of $\mathbf{T}$. Then there exist $\mathbb{N}_{0} \in \mathbb{N}$ and a subsequence $\left\{\omega_{n_{r}}\right\}$ of $\left\{\omega_{n}\right\}$ such that $d_{\sigma}\left(\omega_{2 n_{r}},[\mathbf{T} \rho]_{\alpha_{\mathbf{T}}(\rho)}\right)>0$ for all $n_{r} \geq \mathbb{N}_{0}$. As $d_{\sigma}\left(\omega_{2 n_{r}},[\mathbf{T} \rho]_{\alpha_{\mathbf{T}}(\rho)}\right)>0$ for all $n_{r} \geq \mathbb{N}_{0}$, from Lemma 2.1, condition (1) of Definition 1.3, and (6) we have

$$
\begin{aligned}
& \Omega+\mathbf{F}\left(d_{\sigma}\left(\omega_{2 n_{r}},[\mathbf{T} \rho]_{\alpha_{\mathbf{T}}(\rho)}\right)\right) \\
& \leq \Omega+\mathbf{F}\left(H_{\sigma}\left(\left[\mathbf{S} \omega_{2 n_{r}-1}\right]_{\alpha_{\mathbf{S}}\left(\omega_{2 n_{r}-1}\right)},[\mathbf{T} \rho]_{\alpha_{\mathbf{T}}(\rho)}\right)\right) \\
& \leq \mathbf{F}\left(d_{\sigma}\left(\omega_{2 n_{r}-1}, \rho\right)\right)+€ \min \left\{d_{\sigma}\left(\omega_{2 n_{r}-1},\left[\mathbf{S} \omega_{2 n_{r}-1}\right]_{\alpha_{\mathbf{S}}\left(\omega_{2 n_{r}-1}\right)}\right),\right. \\
& \left.d_{\sigma}\left(\rho,[\mathbf{T} \rho]_{\alpha_{\mathbf{T}}(\rho)}\right), d_{\sigma}\left(\omega_{2 n_{r}-1},[\mathbf{T} \rho]_{\alpha_{\mathbf{T}}(\rho)}\right), d_{\sigma}\left(\rho,\left[\mathbf{S} \omega_{2 n_{r}-1}\right]_{\alpha_{\mathbf{S}}\left(\omega_{2 n_{r}-1}\right)}\right)\right\} \\
& \leq \mathbf{F}\left(d_{\sigma}\left(\omega_{2 n_{r}-1}, \rho\right)+\succeq \min \left\{d_{\sigma}\left(\omega_{2 n_{r}-1}, \omega_{2 n_{r}}\right), d_{\sigma}\left(\rho,[\mathbf{T} \rho]_{\alpha_{\mathbf{T}}(\rho)}\right),\right.\right. \\
& \left.d_{\sigma}\left(\omega_{2 n_{r}-1},[\mathbf{T} \rho]_{\alpha_{\mathbf{T}}(\rho)}\right), d_{\sigma}\left(\rho, \omega_{2 n_{r}}\right)\right\}
\end{aligned}
$$

This implies that

$$
\begin{aligned}
\mathbf{F}\left(d_{\sigma}\left(\omega_{2 n_{r}},[\mathbf{T} \rho]_{\alpha_{\mathbf{T}}(\rho)}\right)\right) \leq & \mathbf{F}\left(d_{\sigma}\left(\omega_{2 n_{r}-1}, \rho\right)\right)+\succeq \min \left\{d_{\sigma}\left(\omega_{2 n_{r}-1}, \omega_{2 n_{r}}\right), d_{\sigma}\left(\rho,[\mathbf{T} \rho]_{\alpha_{\mathbf{T}}(\rho)}\right),\right. \\
& \left.d_{\sigma}\left(\omega_{2 n_{r}-1},[\mathbf{T} \rho]_{\alpha_{\mathbf{T}}(\rho)}\right), d_{\sigma}\left(\rho, \omega_{2 n_{r}}\right)\right\}-\Omega \\
< & \mathbf{F}\left(d_{\sigma}\left(\omega_{2 n_{r}-1}, \rho\right)+\succeq \min \left\{d_{\sigma}\left(\omega_{2 n_{r}-1}, \omega_{2 n_{r}}\right), d_{\sigma}\left(\rho,[\mathbf{T} \rho]_{\alpha_{\mathbf{T}}(\rho)}\right),\right.\right. \\
& \left.d_{\sigma}\left(\omega_{2 n_{r}-1},[\mathbf{T} \rho]_{\alpha_{\mathbf{T}}(\rho)}\right), d_{\sigma}\left(\rho, \omega_{2 n_{r}}\right)\right\} .
\end{aligned}
$$

As $\mathbf{F}$ is strictly increasing, we have

$$
\begin{aligned}
d_{\sigma}\left(\omega_{2 n_{r}},[\mathbf{T} \rho]_{\alpha_{\mathbf{T}}(\rho)}\right)< & d_{\sigma}\left(\omega_{2 n_{r}-1}, \rho\right)+\succeq \min \left\{d_{\sigma}\left(\omega_{2 n_{r}-1}, \omega_{2 n_{r}}\right), d_{\sigma}\left(\rho,[\mathbf{T} \rho]_{\alpha_{\mathbf{T}}(\rho)}\right),\right. \\
& \left.d_{\sigma}\left(\omega_{2 n_{r}-1},[\mathbf{T} \rho]_{\alpha_{\mathbf{T}}(\rho)}\right), d_{\sigma}\left(\rho, \omega_{2 n_{r}}\right)\right\} .
\end{aligned}
$$


By taking the limit as $n \rightarrow \infty$ we get

$$
d_{\sigma}\left(\rho,[\mathbf{T} \rho]_{\alpha_{\mathbf{T}}(\rho)}\right) \leq 0
$$

Thus $\rho \in[\mathbf{T} \rho]_{\alpha_{\mathbf{T}}(\rho)}$. By a similar procedure we can prove that $\rho \in[\mathbf{S} \rho]_{\alpha_{\mathbf{S}}(\rho)}$. Hence $\rho \in$ $[\mathbf{T} \rho]_{\alpha_{\mathbf{T}}(\rho)} \cap[\mathbf{S} \rho]_{\alpha_{\mathbf{S}}(\rho)}$.

Theorem 2.2 Let $\left(\mathcal{W}, d_{\sigma}\right)$ be a complete controlled metric space, and let $\mathbf{S}, \mathbf{T}$ be fuzzy mappings from $\mathcal{W}$ into $\Gamma(\mathcal{W})$. Suppose that for each $\omega_{1} \in \mathcal{W}$, there exist $\alpha_{\mathbf{S}}\left(\omega_{1}\right), \alpha_{\mathbf{T}}(y) \in$ $(0,1]$ such that $\left[\mathbf{S} \omega_{1}\right]_{\alpha_{\mathbf{S}}\left(\omega_{1}\right)},[\mathbf{T} y]_{\alpha_{\mathbf{T}}(y)}$ are nonempty closed subsets of $\mathcal{W}$. If there exist $\mathbf{F} \in \mathcal{F}$ and $\Omega>0$ such that

$$
\Omega+\mathbf{F}\left(H_{\sigma}\left(\left[\mathbf{S} \omega_{1}\right]_{\alpha_{\mathbf{S}}\left(\omega_{1}\right)},\left[\mathbf{T} \omega_{2}\right]_{\alpha_{\mathbf{T}}\left(\omega_{2}\right)}\right) \leq \mathbf{F}\left(d_{\sigma}\left(\omega_{1}, \omega_{2}\right)\right)\right.
$$

for all $\omega_{1}, \omega_{2} \in \mathcal{W}$ with $H_{\sigma}\left(\left[\mathbf{S} \omega_{1}\right]_{\alpha_{\mathbf{S}}\left(\omega_{1}\right)},\left[\mathbf{T} \omega_{2}\right]_{\alpha_{\mathbf{T}}\left(\omega_{2}\right)}\right)>0$, then there exists a common $\alpha$-fuzzy fixed point of $\mathbf{S}$ and $\mathbf{T}$.

Proof By taking $€=0$ in Theorem 2.1 we get the proof.

Corollary 2.1 Let $\left(\mathcal{W}, d_{\sigma}\right)$ be a complete controlled metric space, and let $\mathbf{T}$ be a fuzzy mapping from $\mathcal{W}$ into $\Gamma(\mathcal{W})$. Suppose that for each $\omega_{1} \in \mathcal{W}$, there exist $\alpha_{\mathbf{T}}\left(\omega_{1}\right), \alpha_{\mathbf{T}}\left(\omega_{2}\right) \in$ $(0,1]$ such that $\left[\mathbf{T} \omega_{1}\right]_{\alpha_{\mathbf{T}}\left(\omega_{1}\right)},\left[\mathbf{T} \omega_{2}\right]_{\alpha_{\mathbf{T}}\left(\omega_{2}\right)}$ are nonempty closed subsets of $\mathcal{W}$. If there exist $\mathbf{F} \in \mathcal{F}, \Omega>0$, and $€ \geq 0$ such that

$$
\Omega+\mathbf{F}\left(H_{\sigma}\left(\left[\mathbf{T} \omega_{1}\right]_{\alpha_{\mathbf{T}}\left(\omega_{1}\right)},\left[\mathbf{T} \omega_{2}\right]_{\alpha_{\mathbf{T}}\left(\omega_{2}\right)}\right) \leq \mathbf{F}\left(d_{\sigma}\left(\omega_{1}, \omega_{2}\right)\right)+\longleftarrow\left(M\left(\omega_{1}, \omega_{2}\right)\right)\right.
$$

for all $\omega_{1}, \omega_{2} \in \mathcal{W}$ with $H_{\sigma}\left(\left[\mathbf{T} \omega_{1}\right]_{\alpha_{\mathbf{T}}\left(\omega_{1}\right)},\left[\mathbf{T} \omega_{2}\right]_{\alpha_{\mathbf{T}}\left(\omega_{2}\right)}\right)>0$, where

$$
\begin{aligned}
M\left(\omega_{1}, \omega_{2}\right)= & \min \left\{d_{\sigma}\left(\omega_{1},\left[\mathbf{T} \omega_{1}\right]_{\alpha_{\mathbf{T}}\left(\omega_{1}\right)}\right), d_{\sigma}\left(\omega_{2},\left[\mathbf{T} \omega_{2}\right]_{\alpha_{\mathbf{T}}\left(\omega_{2}\right)}\right),\right. \\
& \left.d_{\sigma}\left(\omega_{1},\left[\mathbf{T} \omega_{2}\right]_{\alpha_{\mathbf{T}}\left(\omega_{2}\right)}\right), d_{\sigma}\left(\omega_{2},\left[\mathbf{T} \omega_{1}\right]_{\alpha_{\mathbf{T}}\left(\omega_{1}\right)}\right)\right\}
\end{aligned}
$$

then there exists an $\alpha$-fuzzy fixed point of $\mathbf{T}$.

Proof By taking $\mathbf{S}=\mathbf{T}$ in Theorem 2.1 we get the proof.

Corollary 2.2 Let $\left(\mathcal{W}, d_{\sigma}\right)$ be a complete controlled metric space, and let $\mathbf{T}$ be a fuzzy mapping from $\mathcal{W}$ into $\Gamma(\mathcal{W})$. Suppose that for each $\omega_{1} \in \mathcal{W}$, there exist $\alpha_{\mathbf{T}}\left(\omega_{1}\right), \alpha_{\mathbf{T}}\left(\omega_{2}\right) \in$ $(0,1]$ such that $\left[\mathbf{T} \omega_{1}\right]_{\alpha_{\mathbf{T}}\left(\omega_{1}\right)}$, $\left[\mathbf{T} \omega_{2}\right]_{\alpha_{\mathbf{T}}\left(\omega_{2}\right)}$ are nonempty closed subsets of $\mathcal{W}$. Assume there exist $\mathbf{F} \in \mathcal{F}$ and $\Omega>0$ such that

$$
\Omega+\mathbf{F}\left(H_{\sigma}\left(\left[\mathbf{T} \omega_{1}\right]_{\alpha_{\mathbf{T}}\left(\omega_{1}\right)},\left[\mathbf{T} \omega_{2}\right]_{\alpha_{\mathbf{T}}\left(\omega_{2}\right)}\right) \leq \mathbf{F}\left(d_{\sigma}\left(\omega_{1}, \omega_{2}\right)\right)\right.
$$

for all $\omega_{1}, \omega_{2} \in \mathcal{W}$ with $H_{\sigma}\left(\left[\mathbf{T} \omega_{1}\right]_{\alpha_{\mathbf{T}}\left(\omega_{1}\right)},\left[\mathbf{T} \omega_{2}\right]_{\alpha_{\mathbf{T}}\left(\omega_{2}\right)}\right)>0$. Then there exists an $\alpha$-fuzzy fixed point of $\mathbf{T}$.

Proof By taking $\mathbf{S}=\mathbf{T}$ and $€=0$ in Theorem 2.1 we get the proof. 


\section{Remark 2.2}

(i) Theorem 2.1 generalizes Theorem 2.1 of [39].

(ii) Theorem 2.2 generalizes Theorem 6 of [40].

(iii) Corollary 2.1 (resp., Corollary 2.2) generalizes Corollary 2.3 (resp., Corollary 2.4) of [39].

Corollary 2.3 Let $\left(\mathcal{W}, d_{\sigma}\right)$ be a complete controlled metric space, and let $\mathbf{A}, \mathbf{B}: \mathcal{W} \rightarrow$ $C L B(\mathcal{W})$ be multivalued mappings. Assume that there exist $\mathbf{F} \in \mathcal{F}, \Omega>0$, and $七 \geq 0$ such that

$$
\Omega+\mathbf{F}\left(H_{\sigma}\left(\mathbf{A} \omega_{1}, \mathbf{B} \omega_{2}\right) \leq \mathbf{F}\left(d_{\sigma}\left(\omega_{1}, \omega_{2}\right)\right)+\longleftarrow\left(M\left(\omega_{1}, \omega_{2}\right)\right)\right.
$$

for all $\omega_{1}, \omega_{2} \in \mathcal{W}$ with $H_{\sigma}\left(\mathbf{A} \omega_{1}, \mathbf{B} \omega_{2}\right)>0$, where

$$
M\left(\omega_{1}, \omega_{2}\right)=\min \left\{d_{\sigma}\left(\omega_{1}, \mathbf{A}\left(\omega_{1}\right)\right), d_{\sigma}\left(\omega_{2}, \mathbf{B}\left(\omega_{2}\right)\right), d_{\sigma}\left(\omega_{1}, \mathbf{B}\left(\omega_{2}\right)\right), d_{\sigma}\left(\omega_{2}, \mathbf{A}\left(\omega_{1}\right)\right)\right\} .
$$

Then there is a common fixed point of $\mathbf{A}$ and $\mathbf{B}$.

Proof Let $\alpha: \mathcal{W} \rightarrow(0,1]$ be an arbitrary mapping and define the mappings $\mathbf{S}, \mathbf{T}: \mathcal{W} \rightarrow$ $\mathbf{F}(\mathcal{W})$ by

$$
\mathbf{S}\left(\omega_{1}\right)(\mathbf{T})= \begin{cases}\alpha & \text { if } \mathbf{T} \in \mathbf{A} \omega_{1}, \\ 0 & \text { if } \mathbf{T} \notin \mathbf{A} \omega_{1},\end{cases}
$$

and

$$
\mathbf{T}\left(\omega_{1}\right)(\mathbf{T})= \begin{cases}\alpha & \text { if } \mathbf{T} \in \mathbf{B} \omega_{1}, \\ 0 & \text { if } \mathbf{T} \notin \mathbf{B} \omega_{1} .\end{cases}
$$

Then we obtain

$$
\begin{aligned}
& {\left[\mathbf{S} \omega_{1}\right]_{\alpha\left(\omega_{1}\right)}=\left\{\mathbf{T}: \mathbf{S}\left(\omega_{1}\right)(\mathbf{T}) \geq \alpha\right\}=\mathbf{A} \omega_{1} \text { and }} \\
& {\left[\mathbf{T} \omega_{1}\right]_{\alpha\left(\omega_{1}\right)}=\left\{\mathbf{T}: \mathbf{T}\left(\omega_{1}\right)(\mathbf{T}) \geq \alpha\right\}=\mathbf{B} \omega_{1} .}
\end{aligned}
$$

Therefore we can apply Theorem 2.1 to get a fixed point $\rho \in \mathcal{W}$ such that

$$
\rho \in[\mathbf{S} \rho]_{\alpha \mathbf{S}(\rho)} \cap[\mathbf{T} \rho]_{\alpha_{\mathbf{T}}(\rho)}=\mathbf{A} \rho \cap \mathbf{B} \rho .
$$

Corollary 2.4 Let $\left(\mathcal{W}, d_{\sigma}\right)$ be a complete controlled metric space, and let $\mathbf{A}, \mathbf{B}: \mathcal{W} \rightarrow$ $C L B(\mathcal{W})$ be multivalued mappings. Assume there exist $\mathbf{F} \in \mathcal{F}$ and $\Omega>0$ such that

$$
\Omega+\mathbf{F}\left(H_{\sigma}\left(\mathbf{A} \omega_{1}, \mathbf{B} \omega_{2}\right) \leq \mathbf{F}\left(d_{\sigma}\left(\omega_{1}, \omega_{2}\right)\right)\right.
$$

for all $\omega_{1}, \omega_{2} \in \mathcal{W}$ with $H_{\sigma}\left(\mathbf{A} \omega_{1}, \mathbf{B} \omega_{2}\right)>0$. Then there exists a common fixed point of $\mathbf{A}$ and $\mathbf{B}$.

Proof It suffices to take $€=0$ in Corollary 2.3. 
Corollary 2.5 Let $\left(\mathcal{W}, d_{\sigma}\right)$ be a complete controlled metric space, and let $\mathbf{A}: \mathcal{W} \rightarrow$ $C L B(\mathcal{W})$ be a multivalued mapping. Assume there exist $\mathbf{F} \in \mathcal{F}, \Omega>0$, and $\mathrm{t} \geq 0$ such that

$$
\Omega+\mathbf{F}\left(H_{\sigma}\left(\mathbf{A} \omega_{1}, \mathbf{A} \omega_{2}\right) \leq \mathbf{F}\left(d_{\sigma}\left(\omega_{1}, \omega_{2}\right)\right)+\longleftarrow\left(M\left(\omega_{1}, \omega_{2}\right)\right)\right.
$$

for all $\omega_{1}, \omega_{2} \in \mathcal{W}$ with $H_{\sigma}\left(\mathbf{A} \omega_{1}, \mathbf{A} \omega_{2}\right)>0$, where

$$
M\left(\omega_{1}, \omega_{2}\right)=\min \left\{d_{\sigma}\left(\omega_{1}, \mathbf{A}\left(\omega_{1}\right)\right), d_{\sigma}\left(\omega_{2}, \mathbf{A}\left(\omega_{2}\right)\right), d_{\sigma}\left(\omega_{1}, \mathbf{A}\left(\omega_{2}\right)\right), d_{\sigma}\left(\omega_{2}, \mathbf{A}\left(\omega_{1}\right)\right)\right\} .
$$

Then there exists a fixed point of $\mathbf{A}$.

Proof Take A = B in Corollary 2.3.

Corollary 2.6 Let $\left(\mathcal{W}, d_{\sigma}\right)$ be a complete controlled metric space, and let $\mathbf{A}: \mathcal{W} \rightarrow$ $C L B(\mathcal{W})$ be a multivalued mapping. Assume there exist $\mathbf{F} \in \mathcal{F}$ and $\Omega>0$ such that

$$
\Omega+\mathbf{F}\left(H_{\sigma}\left(\mathbf{A} \omega_{1}, \mathbf{A} \omega_{2}\right) \leq \mathbf{F}\left(d_{\sigma}\left(\omega_{1}, \omega_{2}\right)\right)\right.
$$

for all $\omega_{1}, \omega_{2} \in \mathcal{W}$ with $H_{\sigma}\left(\mathbf{A} \omega_{1}, \mathbf{A} \omega_{2}\right)>0$. Then there exists a fixed point of $\mathbf{A}$.

Proof Take $\mathbf{A}=\mathbf{B}$ and $€=0$ in Corollary 2.3.

\section{Remark 2.3}

(i) Corollary 2.3 generalizes Corollary 2.5 of [39].

(ii) Corollary 2.4 generalizes Corollary 2.6.

(iii) Corollary 2.5 (resp., Corollary 2.6) generalizes Corollary 2.7 (resp., Corollary 2.8) of [39].

We further suppose that $\hat{\mathbf{T}}$ is a multivalued mapping induced by the fuzzy mapping $\mathbf{T}: \mathcal{W} \rightarrow \Gamma(\mathcal{W})$, that is,

$$
\hat{\mathbf{T}}\left(\omega_{1}\right)(\mathbf{T})=\left\{\mu \in \mathcal{W}: \mathbf{T}\left(\omega_{1}\right)(\mu)=\max _{t \in \mathcal{W}} \mathbf{T}\left(\omega_{1}\right)(t)\right\} .
$$

Lemma 2.2 Let $\left(\mathcal{W}, d_{\sigma}\right)$ be a complete controlled metric space, $\mu \in \mathcal{W}$, and let $\mathbf{T}$ be a fuzzy mapping from $\mathcal{W}$ into $\Gamma(\mathcal{W})$ such that $\hat{\mathbf{T}}\left(\omega_{1}\right)$ is a nonempty compact set for all $\omega_{1} \in \mathcal{W}$. Then $\mu \in \hat{\mathbf{T}}(\mu)$ if and only if

$$
\mathbf{T}(\mu)(\mu) \geq \mathbf{T}(\mu)\left(\omega_{1}\right)
$$

for all $\omega_{1} \in \mathcal{W}$.

Proof Suppose that $\mu \in \hat{\mathbf{T}}(\mu)$. Then

$$
\hat{\mathbf{T}}(\mu)(\mu)=\max _{\omega_{1} \in \mathcal{W}} \mathbf{T}(\mu)\left(\omega_{1}\right) .
$$

This implies that

$$
\hat{\mathbf{T}}(\mu)(\mu) \geq \mathbf{T}(\mu)\left(\omega_{1}\right) \quad \text { for all } \omega_{1} \in \mathcal{W} .
$$


Conversely, suppose that

$$
\hat{\mathbf{T}}(\mu)(\mu) \geq \mathbf{T}(\mu)\left(\omega_{1}\right) \quad \text { for all } \omega_{1} \in \mathcal{W} .
$$

Then by the same steps we can show that $\mu \in \hat{\mathbf{T}}(\mu)$.

Corollary 2.7 Let $\left(\mathcal{W}, d_{\sigma}\right)$ be a complete controlled metric space, and let $\hat{\mathbf{S}}, \hat{\mathbf{T}}: \mathcal{W} \rightarrow \Gamma(\mathcal{W})$ be fuzzy mappings such that for each $\omega_{1} \in \mathcal{W}, \hat{\mathbf{S}}\left(\omega_{1}\right)$ and $\hat{\mathbf{T}}\left(\omega_{1}\right)$ are nonempty closed subsets of $\mathcal{W}$. Assume there exist $\mathbf{F} \in \mathcal{F}, \Omega>0$, and $\mathrm{七} \geq 0$ such that

$$
\Omega+\mathbf{F}\left(H_{\sigma}\left(\hat{\mathbf{S}}\left(\omega_{1}\right), \hat{\mathbf{T}}\left(\omega_{2}\right)\right) \leq \mathbf{F}\left(d_{\sigma}\left(\omega_{1}, \omega_{2}\right)\right)+\succeq\left(M\left(\omega_{1}, \omega_{2}\right)\right)\right.
$$

for all $\omega_{1}, \omega_{2} \in \mathcal{W}$ with $H_{\sigma}\left(\hat{\mathbf{S}}\left(\omega_{1}\right), \hat{\mathbf{T}}\left(\omega_{2}\right)\right)>0$, where

$$
M\left(\omega_{1}, \omega_{2}\right)=\min \left\{d_{\sigma}\left(\omega_{1}, \hat{\mathbf{S}}\left(\omega_{1}\right)\right), d_{\sigma}\left(\omega_{2}, \hat{\mathbf{T}}\left(\omega_{2}\right)\right), d_{\sigma}\left(\omega_{1}, \hat{\mathbf{T}}\left(\omega_{2}\right)\right), d_{\sigma}\left(\omega_{2}, \hat{\mathbf{S}}\left(\omega_{1}\right)\right)\right\}
$$

Then there exists $\mu \in \mathcal{W}$ such that $\mathbf{S}(\mu)(\mu) \geq \mathbf{S}(\mu)\left(\omega_{1}\right)$ and $\mathbf{T}(\mu)(\mu) \geq \mathbf{T}(\mu)\left(\omega_{1}\right)$ for all $\omega_{1} \in \mathcal{W}$.

Proof By Corollary 2.3 there exists $\mu \in \mathcal{W}$ such that $\mu \in \hat{\mathbf{S}}(\mu) \cap \hat{\mathbf{T}}(\mu)$. Then from Lemma 2.2 we get

$$
\mathbf{S}(\mu)(\mu) \geq \mathbf{S}(\mu)\left(\omega_{1}\right) \quad \text { and } \quad \mathbf{T}(\mu)(\mu) \geq \mathbf{T}(\mu)\left(\omega_{1}\right)
$$

for all $\omega_{1} \in \mathcal{W}$.

Example 2.1 Let $\mathcal{W}=[0,1]$. Define $d_{\sigma}: \mathcal{W} \times \mathcal{W} \rightarrow[0, \infty)$ by

$$
d_{\sigma}\left(\omega_{1}, \omega_{2}\right)=\left|\omega_{1}-\omega_{2}\right|
$$

Then $\left(\mathcal{W}, d_{\sigma}\right)$ is a complete controlled metric space, where $\sigma: \mathcal{W} \times \mathcal{W} \rightarrow[1, \infty)$ is defined by

$$
\sigma\left(\omega_{1}, \omega_{2}\right)= \begin{cases}1 & \text { if } \omega_{1}, \omega_{2} \in[0,0.5) \\ \omega_{1}+\omega_{2}+2 & \text { otherwise }\end{cases}
$$

For $\alpha \in[0,1)$ and $\omega_{1} \in \mathcal{W}$, define the mappings $\mathbf{S}, \mathbf{T}: \mathcal{W} \rightarrow \Gamma(\mathcal{W})$ by

$$
\mathbf{S}\left(\omega_{1}\right)(\mathbf{T})= \begin{cases}\alpha & \text { if } 0 \leq \mathbf{T} \leq \frac{\omega_{1}}{50} \\ \frac{\alpha}{2} & \text { if } \frac{\omega_{1}}{50}<\mathbf{T} \leq \frac{\omega_{1}}{40} \\ \frac{\alpha}{3} & \text { if } \frac{\omega_{1}}{40}<\mathbf{T} \leq \frac{\omega_{1}}{30} \\ \frac{\alpha}{4} & \text { if } \frac{\omega_{1}}{30}<\mathbf{T} \leq 1\end{cases}
$$


and

$$
\mathbf{T}\left(\omega_{1}\right)(\mathbf{T})= \begin{cases}\alpha & \text { if } 0 \leq \mathbf{T} \leq \frac{\omega_{1}}{20} \\ \frac{\alpha}{4} & \text { if } \frac{\omega_{1}}{20}<\mathbf{T} \leq \frac{\omega_{1}}{10} \\ \frac{\alpha}{5} & \text { if } \frac{\omega_{1}}{10}<\mathbf{T} \leq \frac{\omega_{1}}{5} \\ \frac{\alpha}{7} & \text { if } \frac{\omega_{1}}{5}<\mathbf{T} \leq 1\end{cases}
$$

so that

$$
\left[\mathbf{S} \omega_{1}\right]_{\alpha_{\mathbf{S}}\left(\omega_{1}\right)}=\left[0, \frac{\omega_{1}}{50}\right] \text { and }\left[\mathbf{T} \omega_{1}\right]_{\alpha_{\mathbf{T}}\left(\omega_{1}\right)}=\left[0, \frac{\omega_{1}}{20}\right]
$$

Let $\mathbf{F}(\mathbf{T})=\ln (\mathbf{T})$. Then there exists $\Omega \in\left(0, \ln \frac{\left|\omega_{2}-\omega_{1}\right|}{\left|\omega_{2}-\frac{\omega_{1}}{2}\right| \frac{1}{50}}\right)$ such that

$$
\Omega+\mathbf{F}\left(H_{\sigma}\left(\left[\mathbf{S} \omega_{1}\right]_{\alpha_{\mathbf{S}}\left(\omega_{1}\right)},\left[\mathbf{T} \omega_{2}\right]_{\alpha_{\mathbf{T}}\left(\omega_{2}\right)}\right) \leq \mathbf{F}\left(d_{\sigma}\left(\omega_{1}, \omega_{2}\right)\right)\right.
$$

for all $\omega_{1}, \omega_{2} \in \mathcal{W}$ with $H_{\sigma}\left(\left[\mathbf{S} \omega_{1}\right]_{\alpha_{\mathbf{S}}\left(\omega_{1}\right)},\left[\mathbf{T} \omega_{2}\right]_{\alpha_{\mathbf{T}}\left(\omega_{2}\right)}\right)>0$. Hence all the axioms of Theorem 2.1 are satisfied, and therefore $0 \in[\mathbf{S} 0]_{\alpha} \cap[\mathbf{T} 0]_{\alpha}$.

\section{Conclusion}

In this work, we introduced the concept of fuzzy mappings in a more general space, called a controlled metric space. Further, we derived the existence of common $\alpha$-fuzzy fixed points for two fuzzy mappings under generalized almost F-contractions in the setting of controlled metric spaces. Our results generalize many well-known results in the literature. For justification of the obtained results, we gave an illustrative example.

\section{Acknowledgements}

The authors are grateful to their Universities for their support.

\section{Funding}

This research did not receive any external funding.

\section{Availability of data and materials}

Not applicable.

\section{Competing interests}

The authors declare that they have no competing interests.

\section{Authors' contributions}

All authors contributed equally and significantly in writing this paper. All authors read and approved the final manuscript.

\section{Author details}

'School of Natural Sciences, National University of Sciences and Technology (NUST), Sector H-12, Islamabad, Pakistan. ${ }^{2} S c h o o l$ of Electrical Engineering and Computer Science (SEECS), National University of Sciences and Technology (NUST), Sector H-12, Islamabad, Pakistan. ${ }^{3}$ Institut Supérieur d'Informatique et des Techniques de Communication, Université de Sousse, H. Sousse 4000, Sousse, Tunisia. ${ }^{4}$ China Medical University Hospital, China Medical University, Taichung, 40402, Taiwan. ${ }^{5}$ Department of Mathematics and Applied Mathematics, Sefako Makgatho Health Sciences University, Ga-Rankuwa, South Africa. ${ }^{6}$ Quantum Leap Africa (QLA), AIMS Rwanda Centre, Remera Sector KN 3, Kigali, Rwanda.

\section{Publisher's Note}

Springer Nature remains neutral with regard to jurisdictional claims in published maps and institutional affiliations. 


\section{References}

1. Banach, S.: Sur les opérations dans les ensembles abstraits et leur application aux équations intégrales. Fundam. Math. 3, 133-181 (1922)

2. Aydi, H., Karapinar, E., Roldan Lopez de Hierro, A.F.: w-interpolative Ćirić-Reich-Rus-type contractions. Mathematics. 7(1), 57 (2019)

3. Jleli, M., Samet, B.: A new generalization of the Banach contraction principle. J. Inequal. Appl. 2014, 38, 1-8 (2014)

4. Aydi, H., Chen, C.M., Karapinar, E.: Interpolative Ćirić-Reich-Rus type contractions via the Branciari distance. Mathematics. 7(1), 84 (2019)

5. Mizoguchi, N., Takahashi, W.: Fixed point theorem for multivalued mappings on complete metric space. J. Math. Anal. Appl. 141, 177-188 (1989)

6. Aydi, H., Jleli, M., Samet, B.: On positive solutions for a fractional thermostat model with a convex-concave source term via $\psi$-Caputo fractional derivative. Mediterr. J. Math. 17(1), 16 (2020)

7. Sintamarian, A.: Integral inclusions of Fredholm type relative to multivalued $\varphi$-contraction. Semin. Fixed Point Theory Cluj-Napoca 3, 361-368 (2002)

8. Berinde, $\mathrm{V}$ : Approximating fixed points of weak contractions using the Picard iteration. Nonlinear Anal. Forum 9 43-53 (2004)

9. Berinde, V.: General constructive fixed point theorems for Ćirić-type almost contractions in metric spaces. Carpath. J. Math. 24, 10-19 (2008)

10. Wardowski, D.: Fixed points of a new type of contractive mappings in complete metric spaces. Fixed Point Theory Appl. 2012, 94 (2012)

11. Ameer, E., Aydi, H., Arshad, M., De la Sen, M.: Hybrid Ćirić type graphic $(\Upsilon, \Lambda)$-contraction mappings with applications to electric circuit and fractional differential equations. Symmetry 12(3), 467 (2020)

12. Piri, H., Kumam, P.: Fixed point theorems for generalized F-Suzuki-contraction mappings in complete $b$-metric spaces Fixed Point Theory Appl. 2016, 90 (2016)

13. Hammad, H.A., Aydi, H., Mlaiki, N.: Contributions of the fixed point technique to solve the 2D Volterra integral equations, Riemann-Liouville fractional integrals, and Atangana-Baleanu integral operators. Adv. Differ. Equ. 2021, 97 (2021)

14. Hammad, H.A., Aydi, H., Gaba, Y.U.: Exciting fixed point results on a novel space with supportive applications. J. Funct. Spaces 2021, Article ID 6613774 (2021)

15. Vetro, F.: F-Contractions of Hardy-Rogers type and application to multistage decision processes. Nonlinear Anal., Model. Control 21(4), 531-546 (2016)

16. Ameer, E., Aydi, H., Arshad, M., Alsamir, H., Noorani, M.S.: Hybrid multivalued type contraction mappings in $\alpha_{K}$-complete partial $b$-metric spaces and applications. Symmetry 11(1), 86 (2019)

17. Altun, I., Minak, G., Dag, H.: Multivalued $\mathbb{F}$-contractions on complete metric spaces. J. Nonlinear Convex Anal. 16, 659-666 (2015)

18. Nadler, S.B.: Multi-valued contraction mappings. Not. Am. Math. Soc. 14, 930 (1967)

19. Kamran, T., Samreen, M., Ain, Q.U.: A generalization of $b$-metric space and some fixed point theorems. Mathematics. 5, $1-7(2017)$

20. Czerwik, S.: Contraction mappings in b-metric spaces. Acta Math. Inform. Univ. Ostrav. 1, 5-11 (1993)

21. Bakhtin, I.A.: The contraction mapping principle in almost metric spaces. Funct. Anal. 30, 26-37 (1989)

22. Alqahtani, B., Fulga, A., Karapinar, E.: Non-unique fixed point results in extended $b$-metric space. Mathematics. 6, 1-11 (2018)

23. Karapinar, E., Czerwik, S., Aydi, H.: $(\alpha, \psi)$-Meir-Keeler contraction mappings in generalized b-metric spaces. J. Funct. Spaces 2018, Article ID 3264620 (2018)

24. Kiran, Q., Alamgir, N., Mlaiki, N., Aydi, H.: On some new fixed point results in complete extended $b$-metric spaces. Mathematics. 7, $476(2019)$

25. Alamgir, N., Kiran, Q., Aydi, H., Mukheimer, A.: A Mizoguchi-Takahashi type fixed point theorem in complete extended b-metric spaces. Mathematics. 7, 478 (2019)

26. Mlaiki, N., Aydi, H., Souayah, N., Abdeljawad, T:: Controlled metric type spaces and the related contraction principle. Mathematics. 6, 194 (2018)

27. Abdeljawad, T., Mlaiki, N., Aydi, H., Souayah, N.: Double controlled metric type spaces and some fixed point results. Mathematics. 6(12), $320(2018)$

28. Alamgir, N., Kiran, Q., Isik, H., Aydi, H.: Fixed point results via a Hausdorff controlled type metric. Adv. Differ. Equ. 2020, 24, 1-20 (2020)

29. Heilpern, S.: Fuzzy mappings and fixed point theorems. J. Math. Anal. Appl. 83, 566-569 (1981)

30. Zadeh, L.Z:: Fuzzy sets. Inf. Control 8, 338-353 (1965)

31. Abbas, M., Damjanović, B., Lazović, R.: Fuzzy common fixed point theorems for generalized contractive mappings. Appl. Math. Lett. 23, 1326-1330 (2010)

32. Ahmad, J., Azam, A., Romaguera, S.: On locally contractive fuzzy set valued mappings. J. Inequal. Appl. 2014, 74 1-10 (2014)

33. Frigon, M., O'Regan, D.: Fuzzy contractive maps and fuzzy fixed points. Fuzzy Sets Syst. 129, 39-45 (2002)

34. Phiangsungnoen, S., Sintunavarat, W., Kumam, P.: Fuzzy fixed point theorems in Hausdorff fuzzy metric spaces. J. Inequal. Appl. 2014, 201, 1-10 (2014)

35. Singh, B., Chauhan, M.S.: Fixed points of associated multimaps of fuzzy maps. Fuzzy Sets Syst. 110, 131-134 (2000)

36. Ahmad, J., Al-Mazrooei, A.E., Aydi, H., De la Sen, M.: On fixed point results in controlled metric spaces. J. Funct. Spaces 2020, 7 (2020)

37. Ahmad, J., Marino, G., Al-Mizel, S.A.: Common fuzzy fixed point results for $\mathbb{F}$-contraction with applications Mathematics. 9, 277 (2021)

38. Al-Mizel, S.A., Ahmad, J., De la Sen, M.: Some new fuzzy fixed point results with applications. Mathematics. 8, 995 (2020)

39. Eqal Al-Mazrooei, A., Ahmad, J.: Fuzzy fixed point results of generalized almost F-contraction. J. Math. Comput. Sci. 2, 206-215 (2018)

40. Ahmad, J., Aydi, H., Mlaiki, N.: Fuzzy fixed points of fuzzy mappings via $\mathbb{F}$-contractions and an application. J. Intell. Fuzzy Syst. 4, 5487-5493 (2019) 